\title{
Pressure-enhanced ferroelectric polarisation in a polar perovskite-like [C2H5NH3]Na0.5Cr0.5(HCOO)3 metal-organic framework
}

Ptak, Maciej; Collings, Ines E.; Svane, Katrine L.; Sieradzki, Adam; Paraguassu, Waldeci; Maczka, Mirosaw

Published in:

Journal of Materials Chemistry C

Link to article, DOI:

$10.1039 / \mathrm{c} 9 \mathrm{tc} 01924 \mathrm{c}$

Publication date:

2019

Document Version

Peer reviewed version

Link back to DTU Orbit

Citation (APA):

Ptak, M., Collings, I. E., Svane, K. L., Sieradzki, A., Paraguassu, W., \& Maczka, M. (2019). Pressure-enhanced ferroelectric polarisation in a polar perovskite-like [C2 $\mathrm{H} 5 \mathrm{NH} 3] \mathrm{Na} 0.5 \mathrm{Cr} 0.5(\mathrm{HCOO}) 3$ metal-organic framework. Journal of Materials Chemistry C, 7(28), 8660-8668. https://doi.org/10.1039/c9tc01924c

\section{General rights}

Copyright and moral rights for the publications made accessible in the public portal are retained by the authors and/or other copyright owners and it is a condition of accessing publications that users recognise and abide by the legal requirements associated with these rights.

- Users may download and print one copy of any publication from the public portal for the purpose of private study or research.

- You may not further distribute the material or use it for any profit-making activity or commercial gain

- You may freely distribute the URL identifying the publication in the public portal 


\section{Pressure-enhanced ferroelectric polarisation in polar perovskite- like $\left[\mathrm{C}_{2} \mathrm{H}_{5} \mathrm{NH}_{3}\right] \mathrm{Na}_{0.5} \mathrm{Cr}_{0.5}(\mathrm{HCOO})_{3}$ metal-organic framework}

Received 00th January 20xx, Accepted 00th January 20xx

DOI: $10.1039 / x 0 x \times 00000 x$

\author{
Maciej Ptak, ${ }^{* a}$ Ines E. Collings, ${ }^{b}$ Katrine L. Svane, ${ }^{\text {cd }}$ Adam Sieradzki, $^{\text {e }}$ Waldeci Paraguassu ${ }^{f}$ and \\ Mirosław Mączka ${ }^{a}$
}

\begin{abstract}
We report the high-pressure structure-related properties of a ferroelectric heterometallic formate perovskite framework templated by the ethylammonium cation $\left(\mathrm{CH}_{3} \mathrm{CH}_{2} \mathrm{NH}_{3}{ }^{+}\right.$, EtA $\left.{ }^{+}\right)$. The high-pressure $X$-ray diffraction studies show a first-order structural phase transition at 3.6(2) GPa from the polar $P n$ ambient phase to a centrosymmetric $P 2_{1} / n$ high-pressure phase. The high-pressure Raman scattering experiment indicates the same transition in the 4.0-4.4 GPa range. The mechanism of the phase transition involves strong compression and distortion of the $\mathrm{NaO}_{6}$ subnetwork and the decrease in the space available for the accommodated $\mathrm{EtA}^{+}$cations resulting in a change in their configuration within the pores at $3.7 \mathrm{GPa}$. Using density functional theory the value of the ferroelectric polarisation within the ac plane is calculated to $0.9 \mu \mathrm{C} / \mathrm{cm}^{2}$ at ambient pressure, increasing in magnitude to a value of $1.1 \mu \mathrm{C} / \mathrm{cm}^{2}$ at a pressure of $3 \mathrm{GPa}$ before vanishing at the phase transition.
\end{abstract}

\section{Introduction}

Ferroelectric materials possess a spontaneous electric polarisation that can be inverted by application of an electric field, and they constitute an important part of many electronic devices. Since ferroelectricity was first discovered in Rochelle salt, ${ }^{1}$ the field has been dominated by inorganic materials such as $\mathrm{BaTiO}_{3}$ and $\mathrm{Pb}\left(\mathrm{Zr}_{\mathrm{x}} \mathrm{Ti}_{1-\mathrm{x}}\right) \mathrm{O}_{3}$, however ferroelectricity is also present in a number of organic and hybrid organic-inorganic materials. ${ }^{2,3}$ While only a few of these materials have characteristics that are comparable with those of inorganic materials, ${ }^{4,5}$ their lightness, flexibility and tunability opens up for a range of new applications of ferroelectrics. This however requires further understanding of how the ferroelectric ordering is influenced by the choice of structural components and how it responds to external stimuli such as temperature and pressure.

\footnotetext{
a. Institute of Low Temperature and Structure Research, Polish Academy of Sciences, Wrocław, Poland.

b. European Synchrotron Radiation Facility, Grenoble, France.

c. Department of Chemistry, University of Bath, Bath, United Kingdom.

d. Department of Energy Conversion and Storage, Technical University of Denmark, Kgs. Lyngby, Denmark.

e. Faculty of Fundamental Problems of Technology, Wrocław University of

Technology, Wrocław, Poland.

f. Faculty of Physics, Federal University of Pará, Belém, Brazil.

+ Corresponding author: M. Ptak,m.ptak@intibs.pl.

Electronic Supplementary Information (ESI) available: Experimental and simulated $\mathrm{X}$-ray diffraction patterns, the crystal structure and visualisation of $\mathrm{HBs}$, calculated and experimental unit cell parameters, the $\mathrm{NH} \cdots \mathrm{O}$ distances as a function of pressure, the $\mathrm{hkO}$ reciprocal space for phases I and II, the two twin domains formed in the HP phase, pictures of crystal in the pressure cell, crystallographic details for both phases, calculated HB parameters in both phases of EtANaCr (pdf). Crystal structures of EtANaCr at all pressures have been deposited to the CCDC with the numbers 1896598-1896610. DFT optimised structures are available from DOI: 10.5281/zenodo.2608304 (link will be activated after acceptance). See DOI: $10.1039 / \times 0 \times x 00000 x$
}

One class of hybrid organic-inorganic materials that have attracted attention due to a number of compounds with ferroelectric and magnetoelectric properties ${ }^{6-17}$ are the formate metal-organic frameworks (MOFs) with perovskitetype topology $\left(\mathrm{AMX}_{3}, \mathrm{X}=\mathrm{HCOO}-\mathrm{M}^{\prime \prime}=\right.$ divalent metal, $\mathrm{A}=$ organic cation, e.g. dimmethylammonium, ethylammonium (EtA+), imidazolium etc.). ${ }^{18}$ The magnetic ordering typically vanishes at low temperatures, while the ferroelectric ordering, which is linked to the configuration of the organic cations within the metal-formate cages, can persist at and above room temperature (RT). ${ }^{19-23}$ The formate perovskites are ideal model systems for studying structure-property relationships due to the many possible combinations of metal and organic cation. In addition, further variation can be achieved by replacing the divalent metal atoms with an equimolar mixture of alkali $\left(\mathrm{M}^{\prime}=\mathrm{Na}^{+}\right.$or $\left.\mathrm{K}^{+}\right)$and trivalent metal ions $\left(\mathrm{M}^{\mathrm{III}}=\mathrm{Fe}^{3+}, \mathrm{Cr}^{3+}\right.$ or $\left.\mathrm{Al}^{3+}\right)$ to form double perovskites with the general formula $\mathrm{AM}^{\prime}{ }_{0.5} \mathrm{M}^{\prime \prime \prime}{ }_{0.5}(\mathrm{HCOO})_{3} .11,22-30$

$\left[\mathrm{C}_{2} \mathrm{H}_{5} \mathrm{NH}_{2}\right] \mathrm{Na}_{0.5} \mathrm{Fe}_{0.5}(\mathrm{HCOO})_{3}$ (EtANaFe) was the first polar and heterometallic formate MOF to be discovered, 22 and is one of the RT ferroelectric formate perovskites. ${ }^{22,31}$ It crystallises in the polar monoclinic Pn space group at low-temperature (LT) and undergoes an order-disorder phase transition at $360 \mathrm{~K}$ to the nonpolar $P 2_{1} / n$ symmetry. In the high-temperature (HT) phase the $\mathrm{EtA}^{+}$cations are dynamically disordered, but in the non-centrosymmetric LT phase the arrangement of the $\mathrm{EtA}^{+}$ cations gives rise to a spontaneous polarisation located in the ac plane. Based on density functional theory (DFT) calculations it was estimated to have a magnitude of $(0.2,0,0.8) \mu \mathrm{C} / \mathrm{cm}^{2} .^{22}$

Further studies of heterometallic compounds with the $\mathrm{EtA}^{+}$ cation showed that replacing $\mathrm{Fe}^{3+}$ ions with $\mathrm{Cr}^{3+}$ (EtANaCr) or $\mathrm{Al}^{3+}$ ions (EtANaAl) retains the crystal structure of both the LT and $\mathrm{HT}$ phases resulting in slight shifts of $\mathrm{T}_{\mathrm{C}}$ to 373 and $369 \mathrm{~K}$, respectively. ${ }^{23}$ The same arrangement of the $\mathrm{EtA}^{+}$cations 
indicates the ferroelectric character of both compounds. In contrast, the substitution of the $\mathrm{Na}^{+}$ions by larger $\mathrm{K}^{+}$ions (EtAKCr) results in a monoclinic structure $\left(P 2_{1} / n\right.$ space group) with ordered $\mathrm{EtA}^{+}$cations which does not undergo any temperature-induced phase transition to a polar space group. ${ }^{26}$ This effect was explained by higher flexibility of the framework composed of the $\mathrm{K}^{+}$ions and the larger voids accommodating the EtA ${ }^{+}$cations. ${ }^{26,32-34}$

Herein, we report the high-pressure structural behaviour of the EtANaCr crystal. The main goal of this work is to define how high pressure influences the polar $P n$ phase and consequently the spontaneous polarisation. Since previous studies have shown that some structural transformations cannot be detected using exclusively X-ray diffraction (XRD) methods, single-crystal diffraction data is combined with Raman scattering to obtain a full profile of the changes in structural properties under high-pressure stimuli. High-pressure XRD, IR and Raman experiments have previously been employed in studies of many formate-based MOFs. ${ }^{27-29,31,35-44}$ However, high-pressure crystal structures have been solved for a few formate frameworks only. ${ }^{35,36,40,42,44}$ Among these compounds non-centrosymmetric high-pressure phases were reported for three crystals only; chiral $\left[\mathrm{NH}_{4}\right] \mathrm{Zn}(\mathrm{HCOO})_{3}$ and perovskite-like $\left[\left(\mathrm{CH}_{3}\right)_{2} \mathrm{NH}_{2}\right] \mathrm{M}^{\prime \prime}(\mathrm{HCOO})_{3}\left(\mathrm{M}^{\prime \prime}=\mathrm{Fe}, \mathrm{Mn}\right){ }^{35,42,44}$ Gomez-Aguirre et al. showed an enhanced ferroelectric polarisation for $\left[\mathrm{NH}_{4}\right] \mathrm{Cd}(\mathrm{HCOO})_{3}$ by SHG measurements at high pressure, but the high-pressure phases were not solved by XRD methods. ${ }^{21}$ The EtANaCr perovskite is therefore the first example of a heterometallic formate MOF with a RT polar and ferroelectric phase investigated under high pressure. To understand how the ferroic properties change during the compression and structural phase transition density functional theory (DFT) calculations were used to estimate the magnitude of the spontaneous polarisation of the EtANaCr crystal. Our results thus give a unique insight into the changes in structural and ferroelectric properties under pressure, which is important for the understanding and further development of hybrid organicinorganic ferroelectrics.

\section{Materials and methods}

\section{Synthesis}

The EtANaCr crystals were synthesized using a solvothermal technique described by us previously. ${ }^{23}$ The phase purity of the bulk sample was confirmed by the good match of their powder XRD patterns with a simulation from the single-crystal structural data CuK $\alpha$ radiation ( $\lambda=1.54056 \AA$ ) (Fig. S1 in Supporting Information) taken from crystal information file (cif) available in literature. ${ }^{23}$

\section{Powder X-ray diffraction}

Powder XRD pattern was obtained on an X'Pert PRO X-ray diffraction system equipped with a PIXcel ultrafast line detector and Soller slits for CuK $\alpha 1$ radiation ( $\lambda=1.54056 \AA$ ). The powders were measured in the reflection mode, and the $\mathrm{X}$-ray tube settings were $30 \mathrm{~mA}$ and $40 \mathrm{kV}$.

\section{High-pressure single-crystal X-ray diffraction}

High-pressure single-crystal X-ray diffraction on the EtANaCr formate was measured at the ID15B beamline of the European Synchrotron Radiation Facility, Grenoble up to $6 \mathrm{GPa}$ using monochromatic X-ray radiation $(\lambda=0.411189 \AA$ Å). Membrane driven LeToullec type diamond anvil cells were used, equipped with Boehler-Almax anvils. Stainless steel was used as the gasket material, and $\mathrm{Ne}$ was loaded as the pressuretransmitting medium (PTM). Diffraction patterns were collected with a Mar555 flat panel detector using steps of $0.5^{\circ}$ oscillations over a total $\omega$ scan range of $76^{\circ}$ about the vertical axis. The pressures were measured using the ruby fluorescence method before and after each diffraction measurement. The average of both pressure values was used and the variance was employed to estimate the errors associated with the pressure measurement, in addition to the \pm 0.05 error to account for the error in the ruby florescence method in the pressure range below $6 \mathrm{GPa}$. Lattice parameter determination and integration of the reflection intensities were performed using the CrysAlisPro software. ${ }^{45}$

Three crystals were selected and positioned in the gasket chamber in order to increase the likelihood of solving potential high-pressure phases. A small piece of tungsten was also loaded alongside the crystals for centring purposes.

\section{High-pressure Raman scattering}

The high-pressure Raman spectra were recorded in backscattering geometry using a microscope attached to a triplegrating spectrometer Jobin-Yvon T64000. The $514.5 \mathrm{~nm}$ line of a solid-state ion laser was used as excitation and the spectral resolution was $2 \mathrm{~cm}^{-1}$. In order to reach high pressures, a diamond anvil cell Diacell ${ }^{\circledast} \mu$ ScopeDAC-RT(G) from Almax easyLab with a diamond of $0.4 \mathrm{~mm}$ of culets was used. The sample was loaded into a $100 \mathrm{~mm}$ hole drilled in a stainless steel gasket with a thickness of $200 \mathrm{~mm}$ using an electric discharge machine from Almax easyLab. The nujol (mineral oil) served as the PTM. Pressures were measured based on the shifts of the ruby $R_{1}$ and $R_{2}$ fluorescence lines.

\section{Density functional theory (DFT) calculations}

Spin-polarised density functional theory (DFT) calculations were performed using the Vienna ab initio simulation package $\left(\right.$ VASP) ${ }^{46}$ and projector augmented wave (PAW) pseudopotentials. The exchange and correlation energy was described using the PBEsol functional ${ }^{47}$ with the D3 correction 48,49 to include the effect of van der Waals interactions. The atomic positions were optimized with an energy cutoff of $700 \mathrm{eV}$ and a $2 \times 2 \times 2 \mathrm{k}$-point mesh until all forces were below $0.01 \mathrm{eV} / \AA ̊$. Calculations for the related compound EtAKCr showed a very small energy difference ( $1 \mathrm{meV})$ between the ferromagnetic and antiferromagnetic alignment of the spin on the $\mathrm{Cr}^{3+}$ ions, and we thus choose to optimize all structures in the ferromagnetic configuration in the following.

The experimentally determined crystal structure was scaled to different volumes and optimized, allowing the relative length 

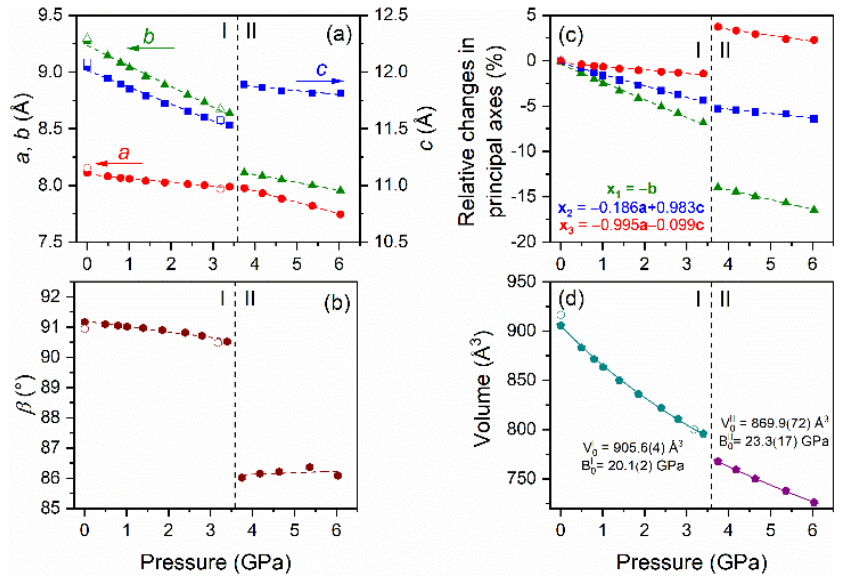

Fig. 1 The changes of the lattice $a, b$ and $c$ parameters (a), monoclinic $B$ angle (b), relative changes in the principal axes (c) and volume (d) with the increasing pressure. The principal axes $\mathbf{x}_{1}, \mathbf{x}_{\mathbf{2}}$ and $\mathbf{x}_{\mathbf{3}}$ were calculated using PASCal. ${ }^{53}$ The volume vs. pressure function for both phases was fitted using a second-order Birch-Murnaghan equation-of-state using EoSFit ${ }^{54}$ and is presented in (d) as a solid lines. The vertical dotted lines present the pressure, where the structural phase transition takes place. The open symbols are values obtained upon decompression.

and angle between unit cell vectors to change while keeping the volume constant. The

spontaneous polarisation density was calculated for each volume using the Berry phase formalism. ${ }^{50}$ In a physical experiment only differences in polarisation are measured as a flow of charges between the surfaces of a crystal. Likewise, it is only physically meaningful to calculate the difference in polarisation between two structures, which must be adiabatically connected along a continuous path. The polarisation difference calculated along this path is independent of the choice of unit cell, provided that it is corrected for sudden jumps, known as polarisation quanta, which arise when electrons or ions move across the periodic boundaries. ${ }^{51,52}$ We therefore create a number of structures along a path connecting the optimized structure with polarisation $\boldsymbol{P}_{\boldsymbol{s}}$ with its mirror image with polarisation $-\boldsymbol{P}_{\boldsymbol{s}}$, corresponding to the switching of the polarisation direction. The difference in polarisation between the end points, $2 \boldsymbol{P}_{\boldsymbol{s}}$, is obtained by calculating the polarisation for each structure along the path, and correcting for any sudden jumps, corresponding to a quantum of polarisation, that occur (c.f. ESI for plots of the polarisation along the path). The total magnitude of the polarisation can then be found as $P_{\text {tot }}=\left|\boldsymbol{P}_{\mathrm{s}}\right|$.

The coordinates of a structure along the path $(\boldsymbol{r})$ can in principle be constructed as a linear combination of the coordinates of the initial and final structures, $\boldsymbol{r}_{P_{S}}$ and $\boldsymbol{r}_{-P_{S}}$

$\boldsymbol{r}=\lambda \boldsymbol{r}_{P_{S}}+(1-\lambda) \boldsymbol{r}_{-P_{S}}$,

where $\lambda$ is a parameter between 0 and 1 . However, since the transition in our case involved a rotation of the molecules, equation 1 was used for the coordinates of the metal formate cage only, while the coordinates of the molecules were changed by a gradual rotation.

\section{Results and discussion}
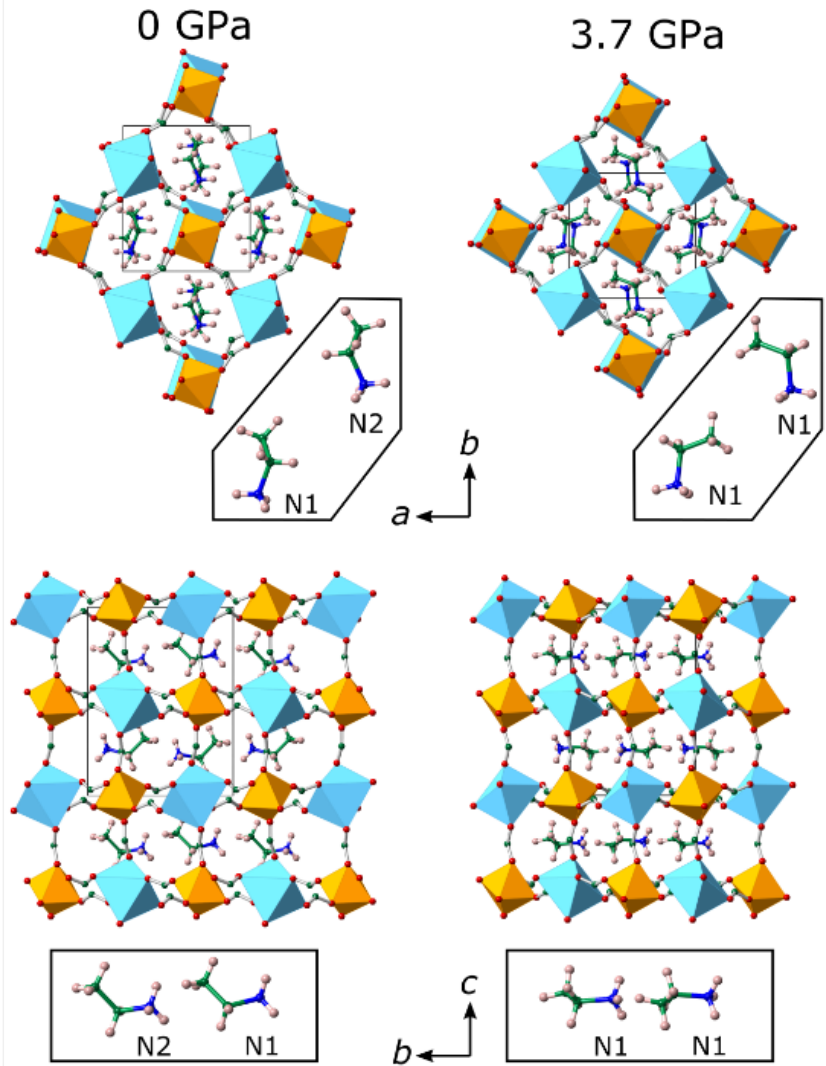

Fig. 2 Structures of EtANaCr at ambient and in the high-pressure phase. Insets in the black boxes show the two symmetry unique $\mathrm{EtA}^{+}$cations for the ambient phase and the equivalent $\mathrm{EtA}+$ cations for the $\mathrm{HP}$ phase. The $\mathrm{CrO}_{6}$ and $\mathrm{NaO}_{6}$ octahedra are shown in orange and light blue, respectively. The colours represent the atoms as red for $\mathrm{O}$, green for $\mathrm{C}$, dark blue for $\mathrm{N}$, and light pink for $\mathrm{H}$. The hydrogen atoms of the formate linkers have been omitted for clarity.

\section{High-pressure single-crystal X-ray diffraction}

The ambient pressure phase (phase I) of ethylammonium sodium-chromium formate (EtANaCr) has previously been refined in the space group $P n$ with the cell parameters $a=8.10474(9) \AA$. $b=9.26392(11) \AA, c=12.04593(14) \AA$ and $B=91.1766(10)^{\circ} .23$ The ordered polar $P n$ phase is in principle ferroelectric as it was calculated for isostructural EtANaFe. ${ }^{22} \mathrm{At}$ $373 \mathrm{~K}$ the order-disorder phase transition takes place and the EtANaCr crystal transforms to the $P 2_{1} / n$ monoclinic symmetry with the cell

parameters $a=8.1066(6) \AA, b=9.3455(11) \AA, c=12.1283(13) \AA$ and $B=91.062(8)^{\circ}$ at $400 \mathrm{~K} .{ }^{23}$

To understand the high-pressure behaviour of the unit cell compression in the monoclinic $P n$ phase, the pressure dependence of the unit cell parameters are plotted in Fig. 1. They all show an abrupt change at about 3.7 GPa due to the occurrence of a first order structural phase transition.

In order to obtain the unique behaviour of the unit cell compression in the ambient polar monoclinic Pn phase, the pressure dependences of the principal axes were calculated using PASCal. ${ }^{53}$ The principal axes $\mathbf{x}_{1}, \mathbf{x}_{2}$ and $\mathbf{x}_{3}$ are related to the lattice parameters by the following relations: $\mathbf{x}_{\mathbf{1}}=-\mathbf{b}, \mathbf{x}_{\mathbf{2}}=-$ $0.186 a+0.983 c$ and $x_{3}=-0.995 a-0.099 c$. There is some degree of 


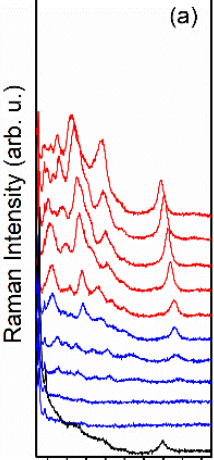

100200300400
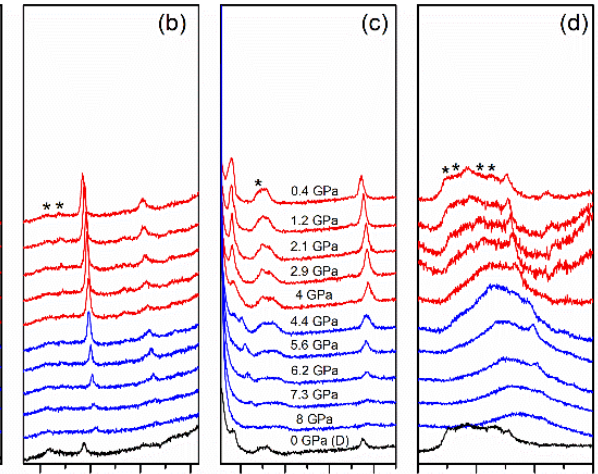

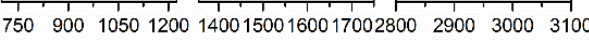
Wavenumber $\left(\mathrm{cm}^{-1}\right)$
Fig. 3 The pressure evolution of Raman spectra for the EtANaCr crystal in phase I (blue colour) and II (red colour).
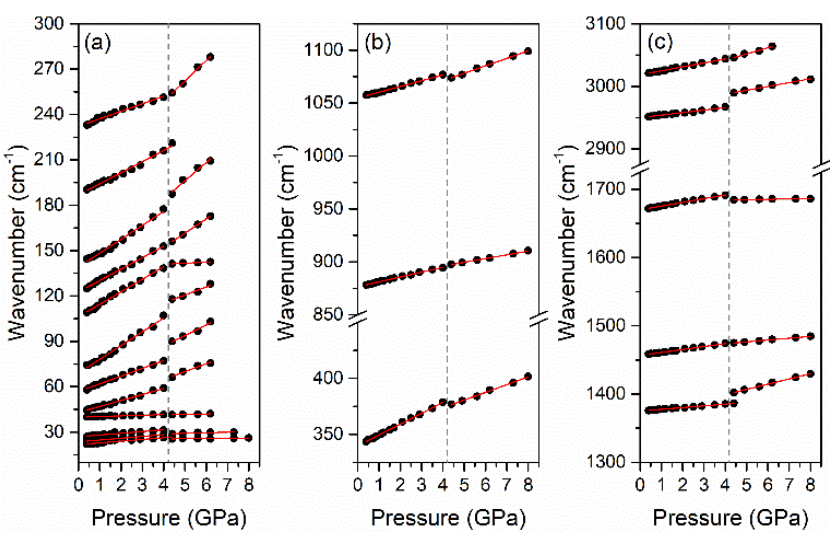

Fig. 4 The pressure-dependence of Raman wavenumbers as a function of pressure. The vertical lines present the pressure, where the structural phase transition takes place.

anisotropy in the compressibility ( $\mathrm{K}$ ) of the principal axes, as $\mathbf{x}_{1}>\mathbf{x}_{2}>\mathbf{x}_{3}$, with $\mathbf{x}_{1}$ reaching up to $\sim 7 \%$ compression (unit cell volume compression is at $12.1 \%$ ) before the phase transition. This anisotropy likely arises from the directionality of the hydrogen bonding (HB) interactions from the $\mathrm{Et}^{+}$to the oxygen atoms of the formate linkers (Fig. S2), which are mainly located within the ac-plane. The skeleton of the $\mathrm{EtA}^{+}$cation lies almost parallel to the $\mathbf{x}_{\mathbf{1}}$ axis, and the space around the alkyl chain, in addition to the lack of direct $\mathrm{H}$-bonds along this direction, make it very compressible compared to the other two directions.

Further examination of the $\mathrm{EtA}^{+}$cations showed that the geometry of the alkyl chains (CC, CN distances and CCN angles) remain almost unaffected even up to the highest applied pressure, $6 \mathrm{GPa}$.

To estimate the compressibility along each axis the data were used to create the compressibility indicatrix using PASCal. ${ }^{53}$ Their main projections showing pressure anisotropy along $\mathbf{x}_{1}, \mathbf{x}_{2}$ and $\mathbf{x}_{3}$ are graphically presented along with the crystal structures and $\mathrm{H}$-bonding (Fig. S2). The obtained $\mathrm{K}_{1}, \mathrm{~K}_{2}$ and $\mathrm{K}_{3}$ coefficients in the 0-3.4 GPa pressure range (referring to the $\mathbf{x}_{1}, \mathbf{x}_{2}$ and $\mathbf{x}_{3}$ principal axes) are 18.4(1) TPa-1, 12.0(2) TPa-1 and 3.4(2) $\mathrm{TPa}^{-1}$, respectively. All coefficients are positive, therefore the EtANaCr crystal exhibits usual pressure behaviour, i.e. positive linear compressibility (PLC) in each direction.
The unit cell volume vs. pressure function was fitted using the second-order Birch-Murnaghen equation-of-state in the pressure range before and after the transition (Fig. 1d). ${ }^{54}$ The fitting for the phase I yields a bulk modulus $B_{0}^{I}=20.1 \pm 0.2 \mathrm{GPa}$ as well as the reference volume $V_{0}^{I}=905.6 \pm 0.4 \AA^{3}$ and in the phase II they are determined as $B_{0}^{I I}=23.3 \pm 1.7 \mathrm{GPa}$ and $V_{0}^{I I}=869.9 \pm 7.1$ $\AA^{3}$. The value of $B_{0}^{I}$ for EtANaCr is lower than the values obtained for the DMAM" (M" $=\mathrm{Cu}$, Fe and $\mathrm{Mn}$ ) family calculated using the same methodology (24.4-27.2 GPa). ${ }^{44}$ Previous DFT calculations of bulk moduli for the heterometallic DMANaCr (21 $\mathrm{GPa})$ and DMAKCr (17 GPa) also showed lower values than for the $\mathrm{M}^{\prime \prime}$ analogue DMAZn $(30 \mathrm{GPa}){ }^{29}$ This suggests that the inclusion of large alkali atoms, with weaker coordination to the formate linkers, leads to a greater compression.

Increasing the pressure to $3.7 \mathrm{GPa}$ leads to abrupt changes in all unit-cell parameters. In particular, large decreases in the $b$ lattice parameter and $b$ angle, coupled with increases in the $a$ and $c$ lattice parameters, are observed (Tab. S1). This gives rise to an overall decrease of $2.4 \%$ in the unit cell volume associated with the transition. The high-pressure phase could be refined in both the $P n$ and the $P 2_{1} / n$ space group, so the higher centrosymmetric symmetry was chosen (phase II). As a test, DFT calculations were used to optimise the high-pressure structural models refined in $P n$. The resulting optimised structure was found to be nearly identical to its mirror image, and had a significantly lower polarisation than the low-pressure phase (the optimised structure and mirror image are available as supplementary information). This suggests that the structure does indeed change to a centrosymmetric space group. The high-pressure phase showed the occurrence of two twin domains, and both domains were integrated in CrysAlisPro and used to solve the phase with ShelxT. ${ }^{55-57}$ The setting with the monoclinic $8<90^{\circ}$ was chosen as it was the most similar setting to the ambient phase. Fig. 2 shows the ambient and highpressure phases, and highlights that the major structural change lies in the methyl group of the $\mathrm{EtA}^{+}$cation. The $\mathrm{EtA}^{+}$ cation configuration within the pores changes in order to accommodate a narrower pore structure of the metal-formate framework in the high-pressure phase (II). In the ambient phase (I), the pore shapes resemble rhombuses and allow the EtA ${ }^{+}$ cations to align along the $b$-axis. In the high-pressure phase, a herringbone-type pore structure is adopted to reduce void space and forces the alignment of the $\mathrm{EtA}^{+}$cations along the diagonal of the ab-plane.

\section{High-pressure Raman scattering}

To complement the structural properties of the studied polar MOF we performed high pressure Raman scattering experiments. Fig. 3 shows the Raman spectra measured up to 8 GPa. Fig. 4 presents the pressure-dependent evolution of the observed Raman bands. The spectrum of EtANaCr measured at the lowest pressure is nearly the same as that obtained outside the pressure chamber. ${ }^{23}$ Some additional bands corresponding to the PTM (mineral oil) are marked with asterisks. It is wellknown that the high-pressure dependence of bands is linear and can be described using a simple $\omega(P)=\omega_{0}+\alpha P$ function described 


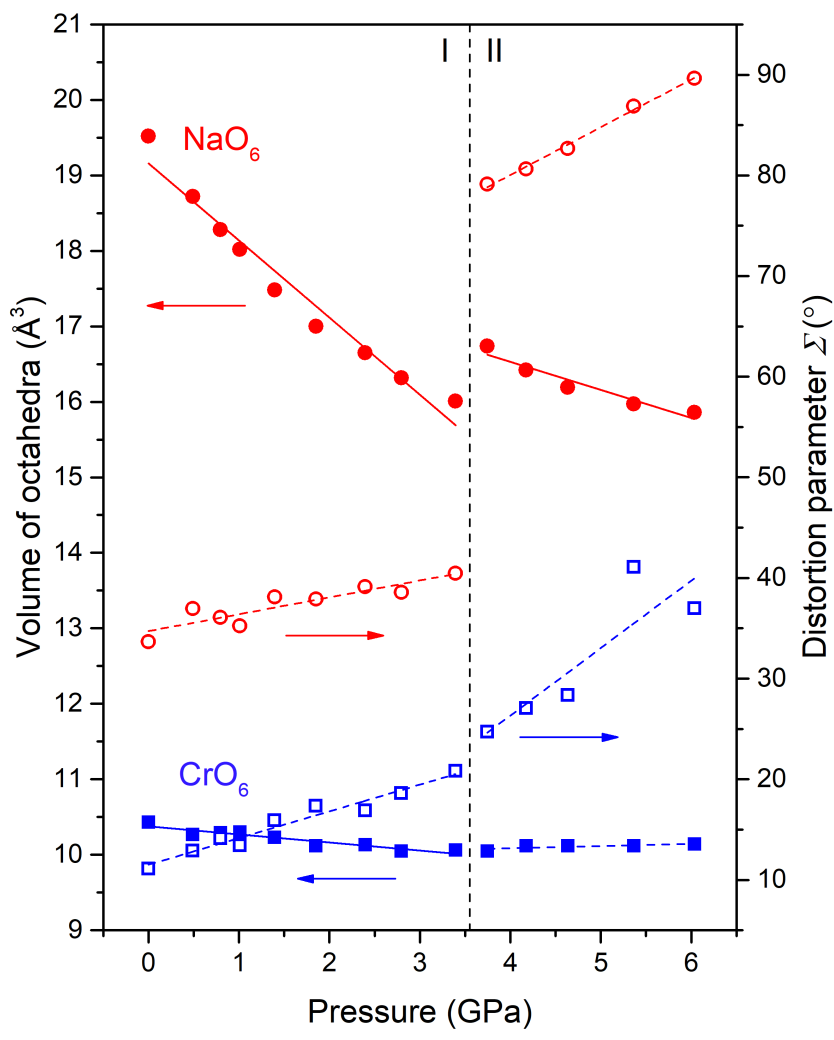

Fig. 5 The $\mathrm{NaO}_{6}$ and $\mathrm{CrO}_{6}$ volume (full symbols) and angle distortion parameter $\Sigma$ (open symbols) as a function of pressure. The dashed vertical line indicates the pressure, where the structural phase transition takes place.

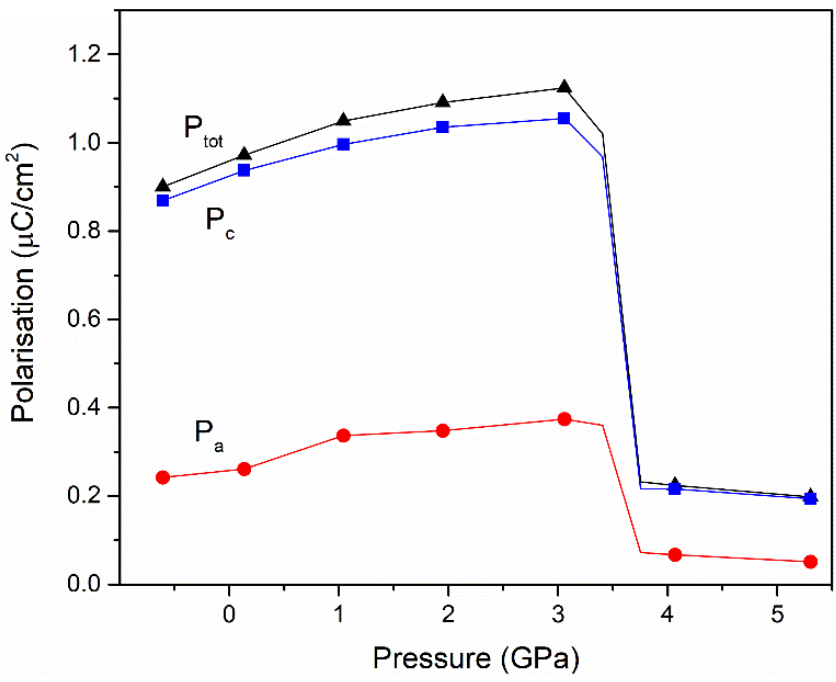

Fig. 6 Polarisation as a function of pressure along the $a$-direction (bottom), the $c$ direction (middle) and total magnitude (top).

by the interception of the function at zero pressure $\left(\omega_{0}\right)$. The second parameter, the so-called vibrational pressure coefficient defined as $\alpha=d \omega / d P$ determine the slope of the curve. The results of the fitting procedure are presented in Fig. 4 while the parameters together with assignments based on the literature data ${ }^{23}$ are summarized in Tab. S2.

Figs. 3 and 4 clearly show that quantitatively the spectra remain unchanged up to $4.0 \mathrm{GPa}$ and a structural phase transition takes place in the 4.0-4.4 GPa region. This phase transition pressure is slightly higher than the one observed from the XRD experiments, most likely due to different PTMs used. In the 4.0-4.4 GPa region, all bands exhibit hardening. The strongest hardening is observed for the translational modes of the $\mathrm{Cr}^{3+}$ ions and lattice modes of the formate ions (Tab. S2). In our previous high-pressure Raman studies of $\mathrm{AmNaCr}$, DMANaCr and ImNaCr, we suggested that such large $\alpha$ parameters for the translational $\mathrm{T}^{\prime}\left(\mathrm{Cr}^{3+}\right)$ modes point to a strong compression of the $\mathrm{CrO}_{6}$ octahedral units. ${ }^{27-29}$ Present highpressure diffraction data, however, allows us to calculate the volume of the $\mathrm{NaO}_{6}$ and $\mathrm{CrO}_{6}$ octahedra as a function of pressure (Fig. 5) using VESTA. ${ }^{58}$ Fig. 5 shows that the volumes of the $\mathrm{CrO}_{6}$ octahedra are almost unaffected in the 0-6 GPa range, while the volumes of the $\mathrm{NaO}_{6}$ octahedra are strongly reduced. This suggests that the large change in wavenumber observed for translational modes of the $\mathrm{Cr}^{3+}$ ions is due to the $\mathrm{Cr}-\mathrm{O}$ bonds being significantly stiffer than the $\mathrm{Na}-\mathrm{O}$ bonds. As a consequence, even a small compression leads to a large change in vibrational frequency, and thereby in wavenumber. The large $\alpha$ coefficients for the lattice modes corresponding mainly to the HCOO- ions (up to $9.19 \mathrm{~cm}^{-1} / \mathrm{GPa}$ ) indicate that continuous compression of the phase I strongly affects the metal-formate framework. The medium values of pressure coefficients observed for modes corresponding to the $\mathrm{EtA}^{+}$cations (4.01$6.36 \mathrm{~cm}^{-1} / \mathrm{GPa}$ ) suggest that they are less affected. The only exceptions are the stretching modes of the methyl and methylene groups (mode 1).

A further increase in pressure to $4.4 \mathrm{GPa}$ leads to some discontinuities in the observed wavenumber shifts and changes in the slope $(\alpha)$ of the wavenumber vs. pressure function. In addition, some lattice modes disappear. This behaviour indicates that EtANaCr undergoes a pressure-induced phase transition in the 4.0-4.4 GPa range. Our spectra show that the observed changes are abrupt, which confirms its first-order nature. In the phase II, the $\alpha$ parameters corresponding to modes associated with translations of the $\mathrm{Cr}^{3+}$ and $\mathrm{Na}^{+}$ions (mode 8 and 9) become lower and higher, respectively. Their high values, together with the increase of the $\alpha$ parameters for lattice modes corresponding to the formate ions (mode 5, 6, 9, 11 and 12), indicate that the metal-formate framework of phase II is also strongly compressible. Large changes in the values of the $\alpha$ coefficients observed for almost all modes corresponding to the internal vibrations of the $\mathrm{EtA}^{+}$cations prove that a rearrangement of $\mathrm{HBs}$ takes place during the phase transition.

The Raman spectrum in the lattice mode region after decompression differs from the one obtained before the highpressure experiment suggesting that the initial ambient phase is not fully recovered. This can be due to a partial amorphization of the sample.

\section{DFT structure optimisation and simulation of high pressure}

To get further information about the structural and ferroelectric properties under pressure, DFT is used to optimise the structure at different volumes. The unit cell parameters of the optimized structure at an external pressure of $0 \mathrm{GPa}$ are compared with the experimental values in Tab. S3. The DFT volume is slightly 
smaller than the experimental volume at ambient pressure, which is partly because the DFT calculations do not include the effect of vibrations at RT. If these effects are included the chosen DFT setup has been shown to give results in very good agreement with experiment for the formate perovskite $\left[\mathrm{NH}_{2} \mathrm{NH}_{3}\right] \mathrm{Zn}(\mathrm{HCOO})_{3} .{ }^{59}$

For each volume the calculated pressure can be used to plot the unit cell parameters as a function of pressure. Since vibrations are not included in the calculation the point of the phase transition cannot be calculated accurately, and we have assumed that it happens when the calculated pressure reaches $3.55 \mathrm{GPa}$. The resulting trends in unit cell parameters closely resembles the experimental results (Fig. S3). Furthermore, the calculated bulk modulus of $28 \mathrm{GPa}$ for phase I, obtained by fitting the energy vs. volume curve to the $2^{\text {nd }}$ order BirchMurnaghan equation-of-state, is in reasonable agreement with the experimentally determined value of $20.3 \mathrm{GPa}$. Calculated bulk modules for hybrid organic-inorganic materials have previously been found to be larger than experimentally determined values, possibly because the calculations neglect effects of temperature and defects. 60

\section{Pressure-dependence of polarisation}

From the symmetry of the $P n$ space group a ferroelectric polarisation is possible along the $a-\left(P_{a} \neq 0\right)$ and $c$-directions $\left(P_{c} \neq 0\right)$, but not along the $b$-direction $\left(P_{b}=0\right)$. For each volume, structures are set up along a path connecting the optimized structure with its mirror image, and the polarisation is calculated along the path using the Berry phase approach (see Experimental section). The calculated value of $P_{\text {tot }}=0.9 \mu \mathrm{C} / \mathrm{cm}^{2}$ (at $0 \mathrm{GPa}$ ) is similar to values that have been calculated for other formate perovskites: $2.6 \mu \mathrm{C} / \mathrm{cm}^{2}$ for $\left[\mathrm{NH}_{2} \mathrm{NH}_{3}\right] \mathrm{Zn}(\mathrm{HCOO})_{3},{ }^{59} 0.82$ $\mu \mathrm{C} / \mathrm{cm}^{2}$ for EtANaFe, ${ }^{22} 1.35 \mu \mathrm{C} / \mathrm{cm}^{2}$ for $\left[\mathrm{NH}_{4}\right] \mathrm{Cd}(\mathrm{HCOO})_{3}{ }^{21} 1.64$ $\mu \mathrm{C} / \mathrm{cm}^{2}$ for EtAMn ${ }^{16}$ and $0.37 \mu \mathrm{C} / \mathrm{cm}^{2}$ for $\left[\mathrm{C}\left(\mathrm{NH}_{2}\right)_{3}\right] \mathrm{Cu}(\mathrm{HCOO})_{3} .{ }^{15}$ For the latter compound the polarisation has also been verified experimentally, revealing a value of $0.11 \mu \mathrm{C} / \mathrm{cm}^{2}{ }^{61}$

Fig. 6 shows the magnitude of the polarisation as a function of pressure as well as the individual components along the $a$ and $c$-axis, assuming that the phase transition occurs at a calculated pressure of $3.55 \mathrm{GPa}$. The plot shows that the polarisation increases with increasing pressure in phase I. The polarisation is defined as $\boldsymbol{P}_{S}=\Delta \mathbf{p} / \mathrm{V}$, where $\Delta \mathbf{p}$ is the dipole moment per unit cell and $\mathrm{V}$ is the unit cell volume. If the dipole moment remains constant under pressure, the smaller unit cell will therefore result in a larger polarisation. The increase in polarisation observed here is however larger than can be explained by the decrease in volume, thus a small increase in the dipole moment must also occur.

At the phase transition the polarisation drops. The points shown for the high-pressure phase in Fig. 6 are calculated by optimising the structure in the $P n$ space group and are shown for comparison of the two possible structural models ( $P n$ vs. $P 2_{1} / n$, the latter having a polarisation of 0 by symmetry). The close resemblance between the $P n$ structure and its mirror image results in a low magnitude of the polarisation. While our results do not conclusively exclude the possibility of a polar high-pressure structure, it is thus clear that the transition to the high pressure phase will lead to a significant drop in the polarisation.

\section{Mechanism of high-pressure structural phase transition}

The XRD results show that the $\mathrm{CrO}_{6}$ units are almost unaffected by the pressure. In phase I they are compressed up to $3.5 \%$ (Fig. 5 ), while their volumes begin to plateau in phase II and their further compression up to $6 \mathrm{GPa}$ is suppressed. A very different behaviour is observed for the $\mathrm{NaO}_{6}$ octahedra, which are more susceptible to the hydrostatic compression. In phase I, their volume decreases by approximately $18.0 \%$. At $3.7 \mathrm{GPa}$, where the phase transition occurs they expand by about $4.6 \%$. Their further compression in phase $I I$ is slower and leads to their continuous contraction to $81.3 \%$ of the zero-pressure volume at $6 \mathrm{GPa}$.

The structural deformation under pressure can also be characterised by the deformation of the $\mathrm{MO}_{6}$ octahedra. The distortion angle parameter $\Sigma$ is defined as:

$\sum=\sum_{i=1}^{12}\left(\left|\phi_{i}-90\right|\right)$,

where $\phi_{i}$ denotes twelve cis angles in the octahedral coordination sphere. ${ }^{24,62}$ Fig. 5 presents the pressuredependence of the calculated $\Sigma$ parameter for each type of octahedra. The $\mathrm{NaO}_{6}$ units are more distorted than the $\mathrm{CrO}_{6}$ ones, but the magnitude of the increasing distortion observed in phase I is very similar for both of them. Above 3.6 GPa there is a dramatic increase in the distortion observed only for the $\mathrm{NaO}_{6}$ units. The rates of distortion in phase II are slightly higher for both types of octahedra. Since both angles, cis and trans, are affected during compression the changes can be described as a tilting and twisting of the octahedra, however this behaviour is more pronounced for the octahedra containing the alkali metal ion.

Finally, changes in the HBs under pressure are considered. Fig. S4 shows that the highest contraction of the unit cell is along the $\mathbf{x}_{\mathbf{1}}$ (b) main axis. All HBs are nearly perpendicular to this direction, which explains why the compression along this direction is favourable. Fig. S4 shows that two independent EtA ${ }^{+}$ cations in the asymmetric unit have different arrangement of $\mathrm{HBs}$ in phase I. Each $\mathrm{H}$-atom on the $\mathrm{NH}_{3}$ group creates two HBs with the formate ions, one having a longer donor-acceptor (D..AA) distance than the other. To evaluate the strength of the $\mathrm{NH} \cdots \mathrm{O}$ interaction, the $\mathrm{HB}$ angle should also be taken into account. As the hydrogen atoms on the $\mathrm{EtA}^{+}$cation were placed based on geometrical and distance constraints, the $\mathrm{N}-\mathrm{H}-\mathrm{O}$ angles were extracted from the DFT optimized structures (Table S4 and S5). These results show that the three shorter bonds ( $D \cdots A<3.0 \AA$ in the $0 \mathrm{GPa}$ structure) have $\mathrm{N}-\mathrm{H}-\mathrm{O}$ angles above $160^{\circ}$, while the three longer bonds ( $\cdots A$ A $>3.0 \AA$ in the $0 \mathrm{GPa}$ structure) have angles below $135^{\circ}$, suggesting that the former are significantly stronger than the latter. The three strong $\mathrm{H}$ bonds shorten under pressure from $\mathrm{O}-\mathrm{H}$ distances in the range of $1.70-1.83 \AA$ at $0 \mathrm{GPa}$ to $1.62-1.77 \AA$ at $3 \mathrm{GPa}$. After the transformation to phase II two of the shorter bonds become longer ( 1.80 and $1.90 \AA$ at $4 \mathrm{GPa})$. This could indicate that the 
structural transformation is related to the $\mathrm{H}$-bonds being compressed beyond their optimal distance in the LP phase.

To summarize, the detailed analysis of structural and Raman data shows that the strong decrease in unit-cell volume observed during compression relates to a decrease in the size of the voids accommodating the $\mathrm{EtA}^{+}$cations and a decrease in the $\mathrm{NaO}_{6}$ octahedral volume. These structural changes allow a small polarization enhancement during the compression. The XRD data show that the CCN geometry (bond lengths and the $\mathrm{CCN}$ angle) remains unaffected upon compression to $6 \mathrm{GPa}$, but its configuration within the pore changes dramatically at the phase transition. The $\mathrm{CH}$ bonds in the methyl and methylene groups are however very sensitive to the change of pressure, as shown by the Raman data, suggesting that they are shortened during the compression.

The XRD data show that the starting structure of the EtANaCr crystal is completely recovered upon decompression from 6 GPa pressure (Fig. 1). The Raman experiment was carried out up to about $8 \mathrm{GPa}$ pressure and does not exhibit similar behaviour upon decompression, but rather suggests a partial decomposition. This could be linked to the loss of hydrostaticity beyond 4-5 GPa for mineral oil, ${ }^{63}$ giving rise to some irreversible changes. The fact that we were not able to fit many of the Raman modes above $6.2 \mathrm{GPa}$, especially in the lattice modes region, supports this conclusion.

\section{Conclusions}

We have investigated the structural and ferroelectric properties of EtANaCr using high-pressure X-ray diffraction combined with high-pressure Raman scattering and density functional theory. The XRD and Raman studies show the occurrence of a first-order structural phase transition, at 3.6 GPa and in the 4.0-4.4 GPa range, respectively. The high-pressure phase was refined in the centrosymmetric polar $P 2_{1} / n$ symmetry, and a detailed mechanism of this phase transition has been proposed. Initially, the increase of pressure results in the strong compression and distortion of the $\mathrm{NaO}_{6}$ subnetwork and the distortion of the $\mathrm{CrO}_{6}$ subnetwork. This causes a decrease in the space available for the accommodated $\mathrm{EtA}^{+}$cations and forces their slight reorientation. At $3.7 \mathrm{GPa}$, where the phase transition occurs, the $\mathrm{EtA}^{+}$cations change their configuration in the pores dramatically. The analysis of the HBs have shown that the two crystallographically independent $\mathrm{EtA}^{+}$cations in the ambientpressure phase become similarly bonded in the high-pressure phase, and that this leads to an elongation of the shortest HBs despite the smaller volume. The partial amorphization is suggested to occur between $6.2 \mathrm{GPa}$ and $8.0 \mathrm{GPa}$ based upon the high-pressure Raman experiment.

DFT calculations show that the $P n$ phase has a ferroelectric polarisation in the ac-plane. Its magnitude is calculated to be $0.9 \mu \mathrm{C} / \mathrm{cm}^{2}$ at $0 \mathrm{GPa}$ and is enhanced upon compression of the low-pressure phase up to $3 \mathrm{GPa}$. The formation of a denser highpressure phase disfavours a polar arrangement of the EtA ${ }^{+}$ cations, and thus the polarisation vanishes at the phase transition.

\section{Conflicts of interest}

There are no conflicts to declare.

\section{Acknowledgements}

This research was supported by the National Science Centre (NCN) in Poland under project No. DEC-2015/17/D/ST5/01339. K. L. S. is funded by ERC programme grant no. 277757 and the Royal Society. We acknowledge computing support from the UK national supercomputing service (Archer), via membership of UK Materials Chemistry Consortium which is funded by EPSRC (EP/L000202). We acknowledge the ESRF for provision of beamtime. I. E. C. thanks J. Jacobs for the Ne gas load. We thank A. Walsh for fruitful discussions.

\section{References}

1 J. Valasek, Phys. Rev., 1921, 17, 475-481.

2 A. S. Tayi, A. Kaeser, M. Matsumoto, T. Aida and S. I. Stupp, Nat. Chem., 2015, 7, 281-294.

3 T. Hang, W. Zhang, H. Y. Ye and R. G. Xiong, Chem. Soc. Rev., 2011, 40, 3577-3598.

4 H.-Y. Ye, Y.-Y. Tang, P.-F. Li, W.-Q. Liao, J.-X. Gao, X.-N. Hua, H. Cai, P.-P. Shi, Y.-M. You and R.-G. Xiong, Science, 2018, 361, 151-155.

5 W.-Q. Liao, D. Zhao, Y.-Y. Tang, Y. Zhang, P.-F. Li, P.-P. Shi, X.-G. Chen, Y.-M. You and R.-G. Xiong, Science, 2019, 363, 1206-1210.

P. Jain, N. S. Dalal, B. H. Toby, H. W. Kroto and A. K. Cheetham, J. Am. Chem. Soc., 2008, 130, 10450-10451. P. Jain, V. Ramachandran, R. J. Clark, H. D. Zhou, B. H. Toby, N. S. Dalal, H. W. Kroto and A. K. Cheetham, J. Am. Chem. Soc., 2009, 131, 13625-13627.

8 M. Mączka, A. Gągor, B. Macalik, A. Pikul, M. Ptak and J. Hanuza, Inorg. Chem., 2014, 53, 457-467.

9 M. Mączka, A. Sieradzki, B. Bondzior, P. Dereń, J. Hanuza and K. Hermanowicz, J. Mater. Chem. C, 2015, 3, 9337-9345.

10 M. Mączka, A. Ciupa, A. Gągor, A. Sieradzki, A. Pikul, B. Macalik and M. Drozd, Inorg. Chem., 2014, 53, 5260-5268.

11 M. Mączka, A. Pietraszko, L. Macalik, A. Sieradzki, J. Trzmiel and A. Pikul, Dalt. Trans., 2014, 43, 17075-17084.

12 B. Pato-Doldán, M. Sánchez-Andújar, L. C. Gómez-Aguirre, S. Yáñez-Vilar, J. López-Beceiro, C. Gracia-Fernández, A. A. Haghighirad, F. Ritter, S. Castro-García and M. A. SeñarísRodríguez, Phys. Chem. Chem. Phys., 2012, 14, 8498-8501.

13 D.-W. Fu, W. Zhang, H.-L. Cai, Y. Zhang, J.-Z. Ge, R.-G. Xiong, S. D. Huang and T. Nakamura, Angew. Chemie Int. Ed., 2011, 50, 11947-11951.

B. Pato-Doldán, L. C. Gómez-Aguirre, J. M. Bermúdez-García, M. Sánchez-Andújar, A. Fondado, J. Mira, S. Castro-García and M. A. Señarís-Rodríguez, RSC Adv., 2013, 3, 2240422411.

A. Stroppa, P. Jain, P. Barone, M. Marsman, J. M. PerezMato, A. K. Cheetham, H. W. Kroto and S. Picozzi, Angew. Chemie Int. Ed., 2011, 50, 5847-5850. 
Soc., 2013, 135, 18126-18130.

R. Shang, G.-C. Xu, Z.-M. Wang and S. Gao, Chem. - A Eur. J., 2014, 20, 1146-1158.

Z. Wang, K. Hu, S. Gao and H. Kobayashi, Adv. Mater., 2010, 22, 1526-1533.

S. Chen, R. Shang, K.-L. Hu, Z.-M. Wang and S. Gao, Inorg. Chem. Front., 2014, 1, 83-98.

F.-F. Wang, C. Chen, Y. Zhang, H.-Y. Ye, Q. Ye and D.-W. Fu, J. Mater. Chem. C, 2015, 3, 6350-6358.

L. C. Gómez-Aguirre, B. Pato-Doldán, A. Stroppa, S. YáñezVilar, L. Bayarjargal, B. Winkler, S. Castro-García, J. Mira, M. Sánchez-Andújar and M. A. Señarís-Rodríguez, Inorg. Chem., 2015, 54, 2109-2116.

M. Ptak, M. M. Mączka, A. Gągor, A. Sieradzki, A. Stroppa, D. Di Sante, J. M. Perez-Mato and L. Macalik, Dalt. Trans., 2016, 45, 2574-2583.

M. Ptak, M. Mączka, A. Gągor, A. Sieradzki, B. Bondzior, P. Dereń and S. Pawlus, Phys. Chem. Chem. Phys., 2016, 18, 29629-29640.

M. Mączka, B. Bondzior, P. Dereń, A. Sieradzki, J. Trzmiel, A. Pietraszko and J. Hanuza, Dalt. Trans., 2015, 44, 6871-6879. A. Sieradzki, J. Trzmiel, M. Ptak and M. Mączka, Electron. Mater. Lett., 2015, 11, 1033-1039.

M. Ptak, A. Gągor, A. Sieradzki, B. Bondzior, P. Dereń, A. Ciupa, M. Trzebiatowska and M. Mączka, Phys. Chem. Chem. Phys., 2017, 19, 12156-12166.

M. Ptak, D. Stefańska, A. Gągor, K. L. Svane, A. Walsh and W. Paraguassu, Phys. Chem. Chem. Phys., 2018, 20, 2228422295.

M. Ptak, B. Zarychta, D. Stefańska, A. Ciupa and W. Paraguassu, Dalt. Trans., 2019, 48, 242-252.

M. Ptak, K. L. Svane, A. Walsh and W. Paraguassu, Phys. Chem. Chem. Phys., 2019, 21, 4200-4208.

Y. Yu, R. Shang, S. Chen, B.-W. Wang, Z.-M. Wang and S. Gao, Chem. - A Eur. J., 2017, 23, 9857-9871.

M. Mączka, A. Gągor, M. Ptak, W. Paraguassu, T. A. Da Silva, A. Sieradzki and A. Pikul, Chem. Mater., 2017, 29, 22642275.

G. Kieslich, S. Kumagai, K. T. Butler, T. Okamura, C. H. Hendon, S. Sun, M. Yamashita, A. Walsh and A. K. Cheetham, Chem. Commun., 2015, 51, 15538-15541.

K. L. Svane, A. C. Forse, C. P. Grey, G. Kieslich, A. K. Cheetham, A. Walsh and K. T. Butler, J. Phys. Chem. Lett., 2017, 8, 6154-6159.

G. Kieslich, S. Sun and A. K. Cheetham, Chem. Sci., 2015, 6 , 3430-3433.

A. V. Chitnis, H. Bhatt, M. Maczka, M. N. Deo and N. Garg, Dalt. Trans., 2018, 47, 12993-13005.

S. Sobczak, A. Chitnis, M. Andrzejewski, M. Maczka, S. Gohil, N. Garg and A. Katrusiak, CrystEngComm, 2018, 20, 53485355.

M. Mączka, P. Kadłubański, P. T. C. Freire, B. Macalik, W. Paraguassu, K. Hermanowicz and J. Hanuza, Inorg. Chem. 2014, 53, 9615-9624.

M. Mączka, N. L. Marinho Costa, A. Gągor, W. Paraguassu, A. Sieradzki and J. Hanuza, 2016, 18, 13993-14000.

M. Viswanathan, CrystEngComm, 2018, 20, 6861-6866.
41

42

W. Li, A. Thirumurugan, P. T. Barton, Z. Lin, S. Henke, H. H.M. Yeung, M. T. Wharmby, E. G. Bithell, C. J. Howard and A. K. Cheetham, J. Am. Chem. Soc., 2014, 136, 7801-7804. M. Viswanathan, Phys. Chem. Chem. Phys., 2018, 20, 1705917070.

W. Li, M. R. Probert, M. Kosa, T. D. Bennett, A. Thirumurugan, R. P. Burwood, M. Parinello, J. A. K. Howard and A. K. Cheetham, J. Am. Chem. Soc., 2012, 134, 1194011943.

M. MącZka, T. Almeida da Silva, W. Paraguassu and K. Pereira Da Silva, Spectrochim. Acta - Part A Mol. Biomol. Spectrosc., 2016, 156, 112-117.

I. E. Collings, M. Bykov, E. Bykova, M. Hanfland, S. Van Smaalen, L. Dubrovinsky and N. Dubrovinskaia, CrystEngComm, 2018, 20, 3512-3521.

Rigaku Oxford Diffraction CrysAlisPro Software System, Version 1.171.39.46, Rigaku. 2018.

G. Kresse and J. Hafner, Phys. Rev. B, 1993, 47, 558-561. J. P. Perdew, A. Ruzsinszky, G. I. Csonka, O. A. Vydrov, G. E. Scuseria, L. A. Constantin, X. Zhou and K. Burke, Phys. Rev. Lett., 2008, 100, 136406.

48 S. Grimme, J. Antony, S. Ehrlich and H. Krieg, J. Chem. Phys., 2010, 132, 154104.

S. Grimme, S. Ehrlich and L. Goerigk, J. Comput. Chem., 2011, 32, 1456-1465.

R. D. King-Smith and D. Vanderbilt, Phys. Rev. B, 1993, 47, 1651-1654.

N. A. Spaldin, J. Solid State Chem., 2012, 195, 2-10.

R. Resta and D. Vanderbilt, Theory of Polarization: A Modern Approach In Physics of Ferroelectrics: A Modern Perspective, pringer: Berlin 2007, pp 31-68.

M. J. Cliffe, A. L. Goodwin J. Appl. Crystallogr., 2012, 45, 1321-1329.

R. J. Angel, M. Alvaro and J. Gonzalez-Platas, Zeitschrift für Krist. - Cryst. Mater., 2014, 229, 405-419. G. M. Sheldrick, Acta Crystallogr. Sect. A Found. Crystallogr., 2008, 64, 112-122.

C. B. Hübschle, G. M. Sheldrick and B. Dittrich, J. Appl. Crystallogr., 2011, 44, 1281-1284.

G. M. Sheldrick, Acta Crystallogr. Sect. A Found. Adv., 2015, 71, 3-8.

K. Momma, F. Izumi J. Appl. Crystallogr., 2011, 44, 12721276.

K. L. Svane and A. Walsh, J. Phys. Chem. C, 2017, 121, 421429.

M. Kosa, J.-C. Tan, C. A. Merrill, M. Krack, A. K. Cheetham and M. Parrinello, ChemPhysChem, 2010, 11, 2332-2336. Y. Tian, A. Stroppa, Y.-S. Chai, P. Barone, M. Perez-Mato, S. Picozzi and Y. Sun, Phys. status solidi - Rapid Res. Lett., 2015, 9, 62-67.

P. Guionneau, M. Marchivie, G. Bravic, J.-F. Létard and D. Chasseau, Springer: Berlin, Heidelberg, pp. 97-128. J. Otto, J. Vassiliou and G. Frommeyer, Phys. Rev. B Condens. Matter Mater. Phys., 1998, 57, 3253-3263. 